\title{
Avaliação dos fatores de risco cardiovascular em motoristas de transporte escolar da Faculdade de Jaguariúna
}

\section{Evaluation of cardiovascular risk factors in school drivers of Jaguariúna Faculty Evaluación de factores de riesgo cardiovascular en conductores de transporte escolar de la Facultad de Jaguariúna}

\author{
Ariene Benatti SILVA ${ }^{\mathbf{1}}$ \\ Bruno Pompeo Bernardes da SILVA ${ }^{1}$ \\ Willian Pires da ROCHA ${ }^{1}$ \\ Marcelo Studart HUNGER ${ }^{2}$ \\ Anderson MARTELLI $\mathbf{I}^{\mathbf{3}}$
}

${ }^{1}$ Graduação em Educação Física pela Faculdade de Jaguariúna FAJ, 13820-000 Jaguariúna-SP, Brasil

${ }^{2}$ Mestre em Performance Humana pela Universidade Metodista de Piracicaba. Docente do Curso de Graduação em Educação Física da FMG - Faculdade Mogiana do Estado de São Paulo UNIMOGI, 13844-070 Mogi Guaçu-SP, Brasil

${ }^{3}$ Mestre; Programa de Pós graduação em Ciências Biomédicas - Centro Universitário Hermínio Ometto, FHO|Uniararas, Araras-SP; Especialista em Laboratório Clínico pela Faculdade de Ciências Médicas-UNICAMP, Campinas-SP. Docente do Curso de Graduação em Educação Física da FMG - Faculdade Mogiana do Estado de São Paulo UNIMOGI, 13844-070 Mogi Guaçu-SP, Brasil

\section{Resumo}

Devido aos inúmeros afazeres do dia a dia e hábito de vida sedentário, os motoristas de vans apresentam inúmeros fatores de riscos para as doenças cardiovasculares (DCV). Este estudo buscou verificar os fatores de riscos para as DCV em motoristas de vans da Faculdade de Jaguariúna (FAJ), município de Jaguariúna-SP que realizam o transporte de alunos. Participaram desta pesquisa 27 motoristas, sendo verificado na amostra pressão arterial, índice de massa corporal (IMC), tabagismo, exercícios físicos, circunferência da cintura e fatores hereditários envolvidos nas DCV. Este estudo possibilitou verificar que essa classe de trabalhadores apresentam fatores de riscos para o desenvolvimento de DCV. Portanto, faz-se necessário, o incentivo de campanhas que visem à prática de atividade física regular, hoje amplamente recomendada, e a conscientização dos motoristas para a adoção de um estilo de vida saudável.

Descritores: Doenças Cardiovasculares; Fatores de Risco; Transportes.

\begin{abstract}
Due to the numerous day-to-day chores and sedentary lifestyle, vans drivers have numerous cardiovascular risk factors (CVD). This study sought to verify the risk factors for CVD in drivers of vans of the Faculty of Jaguariúna (FAJ), municipality of Jaguariúna-SP that carry students. A total of 27 drivers participated in this study. Blood pressure, body mass index (BMI), smoking, physical exercise, waist circumference, and hereditary factors involved in CVD were included in the sample. This study made it possible to verify that this class of workers presents risk factors for the development of CVD. Therefore, it is necessary, the encouragement of campaigns aimed at the practice of regular physical activity, now widely recommended and the awareness of drivers towards the adoption of a healthy lifestyle.

Descriptors: Cardiovascular Diseases; Risk Factors; Transportation
\end{abstract}

\section{Resumen}

Debido a los innumerables que hacer del día a día y hábito de vida sedentario, los conductores de furgonetas presentan numerosos factores de riesgo para las enfermedades cardiovasculares (ECV). Este estudio buscó verificar los factores de riesgo para las ECV en conductores de furgonetas de la Facultad de Jaguariúna (FAJ), municipio de Jaguariúna-SP que realizan el transporte de alumnos. En la muestra, la presión arterial, índice de masa corporal (IMC), tabaquismo, ejercicios físicos, circunferencia de la cintura y factores hereditarios involucrados en las ECV. Este estudio posibilitó verificar que esa clase de trabajadores presentan factores de riesgo para el desarrollo de las ECV. Por lo tanto, se hace necesario, el incentivo de campañas que apunte a la práctica de actividad física regular, hoy ampliamente recomendada y la concientización de los conductores para la adopción de un estilo de vida saludable.

Descriptores: Enfermedades Cardiovasculares; Factores de Riesgo; Transportes.

\section{INTRODUÇÃO}

As condições de vida urbana na atualidade contribuem significativamente para o surgimento de doenças crônicas não transmissíveis (DCNTs) e fazem das doenças cardiovasculares (DCV) um problema da modernidade ${ }^{1,2}$ Essas condições e estilo de vida das pessoas influenciam diretamente um aumento na prevalência de diferentes doenças com redução da expectativa de vida. ${ }^{2}$ Estudos relacionaram a atividade laboral com o aumento do risco cardiovascular, sendo comparado carteiros e seus colegas que desempenhavam funções burocráticas; e motoristas de ônibus em Londres e cobradores que subiam e desciam as escadas dos ônibus de dois andares, sendo observado menor incidência de DCV nos profissionais mais ativos ${ }^{3}$.

A Organização Mundial de Saúde (OMS) aponta que as DCV respondem por aproximadamente 16,7 milhões de óbitos/ano no mundo. No Brasil, calcula-se que as DCV são responsáveis por aproximadamente $30 \%$ dos óbitos em indivíduos acima dos 20 anos de idade $^{4}$ e a hipertensão arterial sistêmica (HAS) participa de quase metade delas ${ }^{5}$. De acordo com a VI Diretrizes Brasileiras de Hipertensão ${ }^{6}$, a mortalidade por doença cardiovascular (DCV) aumenta progressivamente com a elevação da pressão arterial (PA) a partir de $115 / 75 \mathrm{mmHg}$ de forma linear, contínua e independente.
A interação entre diferentes fatores como os ambientais, comportamentais e genéticos pode influenciar o desenvolvimento das DCV, assim, o primeiro passo seria a adoção de estratégias de identificação desses fatores de riscos, visto que a identificação precoce, correção e ações preventivas favorecem a redução das $\mathrm{DCV}^{7}$.

Dessa forma, mostra-se de extrema necessidade protocolos para avaliar de forma simples e rápida a prevalência dos fatores de riscos em populações que exercem diferentes atividades ocupacionais. Nesse contexto, motoristas de transporte urbano, sofrem com estresse, causados pela sobrecarga de trabalho, responsabilidades, altos níveis de concentração requerida, repetitividade de ações ${ }^{8}$, duração da jornada de trabalho, situação precária do asfalto, baixos salários, estado geral dos veículos, ruídos, temperatura, congestionamento, a relação com o público, insegurança com assaltos, sedentarismo e alimentação inadequada, os quais constituem importantes fatores de risco para as $\mathrm{DCV}^{9}$

Assim, o presente estudo apresenta como objetivo investigar os fatores de riscos para a DCV em motoristas de vans que realizam o transporte de alunos para a Faculdade de Jaguariúna (FAJ), localizada no município de Jaguariúna-SP e sua influência na qualidade de vida desses profissionais. 


\section{MATERIAL E MÉTODO}

Trata-se de uma investigação de caráter quantitativo, transversal, observacional e descritivo, realizado seguindo as normas que regulamentam a pesquisa em seres humanos, contidas na Resolução $n^{\circ}$ 466/12 do Conselho Nacional de Saúde, aprovado pelo Comitê de Ética e Pesquisa da Faculdade de Jaguariúna (FAJ) com parecer favorável sob o registro CEP/FAJ: 018/2013.

A amostra foi composta por 27 motoristas, escolhidos de forma aleatória e que transportam regularmente alunos para o campus da FAJ. O recrutamento dos motoristas ocorreu no campus da faculdade e após os esclarecimentos sobre os objetivos e a metodologias da pesquisa, os que concordaram em participar da pesquisa, assinaram o Termo de Consentimento Livre e Esclarecido (TCLE) conforme preconizado na legislação vigente, sendo os mesmos encaminhados para uma sala reservada para a coleta dos dados.

Para a coleta de dados, foi empregada planilha, sendo anotados os dados como: idade, sexo, uso de tabaco, prática de exercícios físicos e fatores hereditários para as DCV. Após esta etapa, a amostra foi submetida à aferição do Índice de Massa corporal - IMC utilizando a metodologia proposta por $\operatorname{Costa}^{10}$, dividindo a massa corporal em quilogramas pelo quadrado da estatura em metros $\left(\mathrm{kg} / \mathrm{m}^{2}\right)$. O equipamento utilizado para medir a estatura dos avaliados foi um estadiômetro da marca Kawee ${ }^{\circledR}$ com precisão de $1 \mathrm{~mm}$ e para a coleta da massa corporal uma balança antropométrica da marca Toledo® com precisão de 100 g e capacidade máxima de $200 \mathrm{Kg}$, aferida e calibrada, garantindo confiabilidade para a realização de tal procedimento. Esse índice expressa a distribuição da massa corporal em relação à estatura e tem sido recomendado para estudos populacionais por sua facilidade de execução.

Para aferição da PA a amostra permaneceu em repouso por 15 minutos absoluto e após esse período, procedeu à verificação da PA utilizando esfigmomanômetro marca Missouri, modelo Aneróide do tipo mecânico e estetoscópio. As medidas de PA foram obtidas no membro superior esquerdo, na posição sentada ${ }^{2}$. Quanto aos valores de PA registrados, a amostra foi classificada segundo os critérios estabelecidos pela VI Diretrizes Brasileira de Hipertensão Arterial $^{6}$.

Para a aferição do perímetro da cintura, foi utilizado a metodologia descrita por Callaway et al. ${ }^{11}$ individuo em pé com abdômen relaxado, os braços ao lado do corpo, sendo posicionado a fita métrica horizontalmente no ponto médio entre a borda inferior da última costela e a crista ilíaca mantendo a fita firme sobre a pele sem esticar excessivamente, evitando-se assim a compressão do tecido subcutâneo.

Como critérios de inclusão da amostra na pesquisa, foram analisadas informações comprobatórias sobre $\mathrm{o}$ transporte constante de alunos para a FAJ desse motorista e o aceite formal em participar da pesquisa com a assinatura do TCLE. Como critério de exclusão, não estar transportando alunos com regularidade à $\mathrm{FAJ}$ e pelo não consentimento em participar da pesquisa.

Após a coleta de dados, as informações foram digitadas e tabuladas em banco de dados utilizando programa Microsoft Excel - 2010 para as análises estatísticas de frequência, média e desvio padrão. Posteriormente foi realizada uma comparação dos resultados observados com os descritos na literatura.

\section{RESULTADOS e DISCUSSÃO}

Foram pesquisados 27 motoristas de vans que realizam o transporte de alunos para o campus da FAJ, no município de Jaguariúna, estado de São Paulo. A totalidade da amostra foi composta homens, com faixa etária entre 32 e 64 anos, média de idade e desvio padrão

$(45,40 \pm 9,60)$.

Um fator de risco para DCV é a utilização de tabaco. Fazendo uso de tabaco, foi observado que quatro motoristas $14,80 \%$ relataram fazer uso. Goodman et al. ${ }^{12}$ destacam que os componentes do tabaco que mais contribuem para os riscos à saúde são o monóxido de carbono, elemento da fase gasosa, associado à nicotina e ao alcatrão, estes substâncias das partículas da fumaça. O consumo do tabaco é um fator de risco para seis das oito causas principais de morte no mundo: doenças cardíacas isquêmicas, acidentes vasculares cerebrais, infecções das vias aéreas inferiores, Doença Pulmonar Obstrutiva Crônica (DPOC), tuberculose e cânceres de pulmão, traqueia e brônquio ${ }^{13}$.

Analisando esses fatores de riscos separadamente, há décadas a relação entre o tabagismo e o risco cardiovascular está bem estabelecida, sendo bem documentadas as evidências, tanto epidemiológicas como metabólicas, das formas como o tabaco contribui para o desenvolvimento da lesão aterosclerótica. $\mathrm{O}$ cigarro aumenta o risco relativo de desenvolver intolerância à glicose e da incidência de diabetes mellitus tipo 2 (DM2), obesidade abdominal, redução de lipoproteínas de alta densidade (HDL), elevação dos triglicerídeos e de lipoproteínas de baixa densidade (LDL). Além disso, o consumo do tabaco eleva a liberação de noradrenalina e adrenalina promovendo a elevação da frequência cardíaca, da resistência vascular periférica e da pressão arterial $^{14}$.

Quanto ao estilo de vida e prevenção da DCV, o sedentarismo se apresenta como um dos principais fatores modificáveis ${ }^{2}$. O exercício físico regular mostra-se como uma estratégia eficaz na redução de patologias clínicas decorrentes do sistema cardiovascular dentre eles a hipertensão arterial sistêmica (HAS) ${ }^{15}$. No presente trabalho foi verificado que $52 \%$ dos entrevistados realizam algum tipo de atividade física (Figura 1). Alquimim et al. ${ }^{9}$ observaram que $50 \%$ da amostra analisada em seu estudo realizava algum tipo de atividade física, corroborando com os dados observados neste estudo. Segundo Matavelli et al. ${ }^{16}$ e Martelli ${ }^{17}$, a prática regular de atividade física é fundamental nas condutas não medicamentosas de prevenção e tratamento da HAS e outras patologias do sistema cardiovascular.

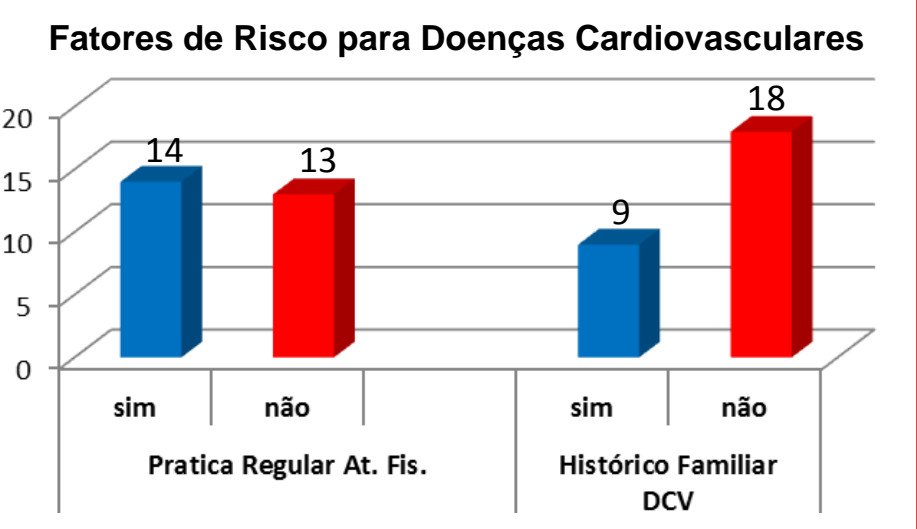

Figura 1: Distribuição dos motoristas de vans que praticam algum tipo de atividade física e histórico familiar de DCV.

Dentre os fatores envolvidos na fisiopatogênese da DCV e da HAS, Barreto-Filho e Krieger ${ }^{18}$ afirmam que um terço deles pode ser atribuído a fatores genéticos. Na amostra pesquisada, foi observado um histórico familiar de DCV em nove motoristas - 33\% (Figura 1). As DCVs são a maior causa de mortalidade nos países desenvolvidos o que faz com que a compreensão da genética cardiovascular seja uma prioridade na investigação médica, uma vez que, já foram descritos uma série de fatores de risco cardiovasculares independentes e, todos apresentando uma ligação forte aos fatores hereditários ${ }^{19}$. 
O excesso de peso e a vida sedentária aparecem como um duplo fator de prevalência para o desenvolvimento da $\mathrm{DCV}^{2}$. Neste sentido, foi verificado o IMC dos motoristas, demonstrando dados preocupantes. Quatro motoristas $(14,80 \%)$ apresentavam peso normal, quinze $(55,50 \%)$ foram classificados como sobrepeso e/ou pré-obeso, quatro $(14,80 \%)$ como obesidade grau I, três motoristas $(11,10 \%)$ obesidade grau II e um motorista $(3,70 \%)$ apresentava obesidade grau III (Figura 2). É possível observar uma parcela significativa de motoristas classificados como sobrepeso.

Esses dados corroboram estudos realizados no Brasil e no exterior ${ }^{8,20}$. Faria et al. ${ }^{21}$ observaram um aumento na prevalência de excesso de peso auto-relatado, sobrepeso e obesidade em 55 condutores de ônibus na cidade de Florianópolis após contratação e início da atividade de motorista, sendo este fator relacionado ao aumento de horas trabalhadas visando maiores rendimentos financeiros, fato que favoreceu o sedentarismo e a redução ao acesso a alimentos saudáveis.

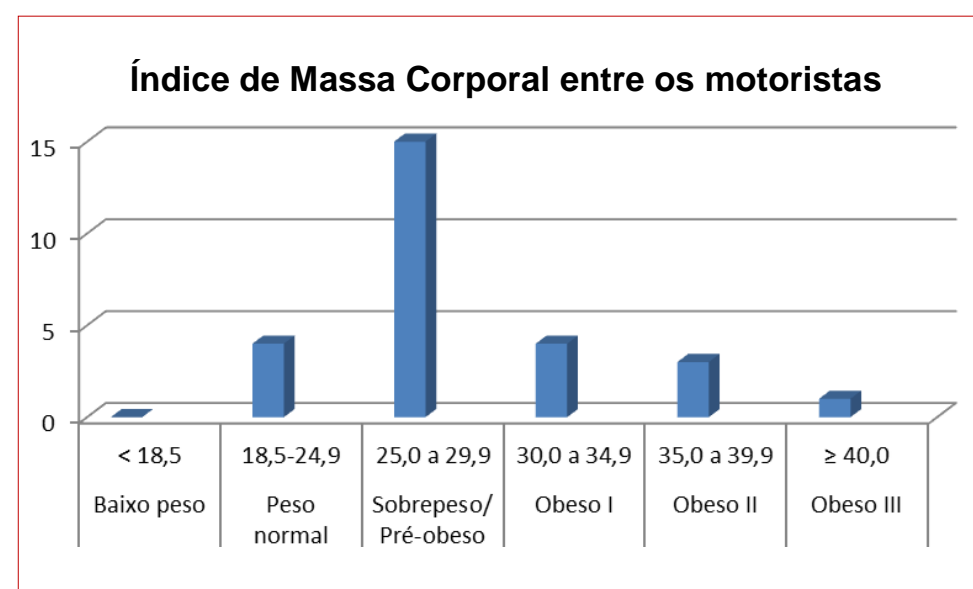

Figura 2: Índice de massa corporal entre os motoristas analisados

Oliveira et al. ${ }^{22}$ apontam que o IMC, apesar de ser mundialmente aceito como um referencial para estabelecer níveis de sobrepeso e obesidade, apresenta vulnerabilidade por não considerar a massa corporal magra, uma questão a ser levada em consideração nos homens, uma vez que a amostra do presente estudo foi composta em sua totalidade por indivíduos do sexo masculino.

Em relação à circunferência abdominal, a amostra apresentou uma média $(102 \mathrm{~cm} \pm 12,0)$, sendo o valor mínimo $79 \mathrm{~cm}$ e valor máximo $129 \mathrm{~cm}$, com $85 \%$ apresentando circunferência abdominal acima do recomendado. Cavagioni et al. ${ }^{23}$ avaliaram 258 caminhoneiros e observaram que $58 \%$ apresentavam circunferência abdominal acima do preconizado. As condições de saúde e estilo de vida observadas no presente trabalho são altamente preocupantes, pois predispõem os sujeitos ao risco de DCV, síndrome metabólica, apneia do sono, entre outras ${ }^{24}$.

A HAS é uma condição clínica multifatorial caracterizada por níveis elevados e sustentados de pressão arterial, com frequentes alterações estruturais e funcionais em determinados órgãos, aumentando consequentemente o risco de eventos cardiovasculares ${ }^{25}$.

Quanto aos resultados dos níveis pressóricos da amostra analisada, esta foi classificada de acordo com a VI Diretrizes Brasileiras de Hipertensão Arterial $^{6}$ como PA ótima $(<120<80)$, normal $(<130<85)$, limítrofe $(130-139$; 85-89), hipertensão estágio 1 (140-159; 90-99), hipertensão estágio 2 (160-179; 100-109) e hipertensão estágio 3 ( $\geq 180$; $\geq 110$ ). Em referência aos níveis pressóricos da amostra total, $11,10 \%(n=3)$ estavam com a PA ótima, $26 \%(n=7)$ pressão normal, $29,60 \% \quad(n=8)$, pressão limítrofe, $11,10 \% \quad(n=3)$ hipertensão estagio $1,18,5(\mathrm{n}=5)$ hipertensão estagio 2 e por fim, 3,70\% ( $\mathrm{n}=1)$, nível 3 de hipertensão de acordo com a classificação da Sociedade Brasileira de Hipertensão (Tabela 1).
Tabela 1. Medidas da aferição da pressão arterial dos motoristas

\begin{tabular}{l|c|c}
\hline \multicolumn{1}{c|}{$\begin{array}{c}\text { Classificação - PA sistólica e } \\
\text { diastólica** }\end{array}$} & $\begin{array}{c}\text { Freq. } \\
\text { Absoluta (n) }\end{array}$ & $\begin{array}{c}\text { Freq. } \\
\text { Relativa (\%) }\end{array}$ \\
\hline Ótima $<120<80$ & 3 & 11,10 \\
Normal $<130<85$ & 7 & 26,00 \\
Limítrofe 130-139 85-89 & 8 & 29,60 \\
Hipertensão estágio 1 & 3 & 11,10 \\
140-159 90-99 & 5 & 18,50 \\
Hipertensão estágio 2 & & \\
160-179 100-109 & 1 & 3,70 \\
Hipertensão estágio 3 $\geq 180 \geq 110$ & $\mathbf{2 7}$ \\
\hline Total & $\mathbf{1 0 0 \%}$ \\
\hline
\end{tabular}

Os dados deste estudo corroboram os resultados descritos por Alquimim et al. ${ }^{9}$ que avaliaram motoristas de transporte urbano de ônibus em Montes Claros (MG) sendo que os principais fatores de riscos foram: sedentarismo, história familiar positiva e aumento da circunferência abdominal. No trabalho de Cavagioni et al. ${ }^{23}$ sobre os fatores de riscos cardiovasculares nos motoristas profissionais de transporte de cargas foram encontrados resultados de alta frequência da HAS, sobrepeso, obesidade e sedentarismo.

Analisando os dados, nota-se que os motoristas apresentam fatores de riscos para a DCV, sendo que o sedentarismo, sobrepeso e circunferência abdominal foram os que mais contribuíram neste estudo para esta classe de doenças. Esses dados apontam para a necessidade de estratégias para a promoção da saúde e a prevenção de doenças crônicas dentre elas DCV nessa profissão, visando modificação do estilo de vida para melhoria da qualidade ${ }^{26}$.

Neri et $a .^{27}$ ao abordar as condições de saúde no setor de transporte rodoviário de cargas e de passageiros, apontam esta categoria como de elevado risco na incidência de DCV, concluindo que é necessário medidas para enfrentar os problemas de saúde colocados devido a atividade do setor, havendo urgência de uma ampliação do debate público sobre as condições de trabalho dessa classe.

\section{CONCLUSÃO}

Com base na metodologia empregada e nos resultados obtidos concluiu-se que os riscos cardiovasculares em motoristas de vans da Faculdade de Jaguariúna (FAJ) podem ser classificados como riscos relevantes para a saúde desses profissionais.

Os fatores que aparecem com maior prevalência neste estudo foram o sedentarismo, inatividade física, sobrepeso e aumento da circunferência abdominal, podendo estar relacionando com a ocupação da amostra estudada. Portanto, faz-se necessário, o incentivo de campanhas que visem à prática de atividades físicas regulares, hoje amplamente recomendadas, além da conscientização dos motoristas para a adoção de estilo de vida mais saudável, uma vez que este profissional está exposto a uma rotina de trabalho desgastante, somada a alimentação pouco saudável, altas responsabilidades quanto ao cumprimento de horários e estresse do trânsito diário.

\section{REFERÊNCIAS}

1. Teixeira ER, Lamas AR, Costa e Silva J, Matos RM. O estilo de vida do cliente com hipertensão arterial e o cuidado com a saúde Esc Anna Nery R Enferm. 2006; 10(3):378-84.

2. Devechio, AP, Pelegrino TA, Silva GRM, Hunger MS, Delbim LR, Magalhães R et al. Fatores que influenciam a hipertensão arterial sistêmica e qualidade de vida em professores universitários. Arch Health Invest. 2017; 6(8):352-58. 
3. Reichert CL. Associação entre fatores metabólicos, antropométricos e clínicos e atividade física em idosos [tese]. Tese doutorado. Porto Alegre: Pontifícia Universidade Católica do Rio Grande do Sul; 2006.

4. Jardim TSV, Jardim PSBV, Araújo WEC, Jardim LMSSV, Salgado CM, et al. Fatores de risco cardiovasculares em coorte de profissionais da área médica: 15 anos de evolução. Arq Bras Cardiol. 2010; 95(3):332-38.

5. Santos ZMSA. Hipertensão arterial: um problema de saúde pública. Rev Bras Promoç Saúde. 2011; 24(4):285-86.

6. IV Diretrizes Brasileiras de Hipertensão - DBH VI. Rev Bras Hipertens. 2010; 17(1):7-10.

7. Alegria E, Cordero A, Laclaustra M, Grima A, León M, Casasnovas JA et al. Prevalencia del síndrome metabólico em población laboral española: registro MESYAS. Rev Esp Cardiol. 2005; 58(7):797-806.

8. Chaves DBR, Costa AGS, Oliveira ARS, Oliveira TC, Araujo TL, Lopes MVO. Fatores de risco para hipertensão arterial: investigação em motoristas e cobradores de ônibus. Rev Enferm UERJ. 2008; 16(3):370-76.

9. Alquimim AF, Barral ABCR, Gomes KC, Rezende MC Avaliação dos fatores de risco laborais e físicos para doenças cardiovasculares em motoristas de transporte urbano de ônibus em Montes Claros (MG). Ciênc saúde coletiva. 2012; 17(8):2151-58.

10. Costa RF. Composição corporal: teoria e prática da avaliação. São Paulo: Manole; 2003.

11. Callaway CW, Chumlea WC, Bouchard C, Himes $\mathrm{JH}$, Lohman TG, Martin AD et al. Circumferences. In Lohman TG, Roche AF, Martorell R (ed.). Anthropometric standardization reference manual. Champaign: Human Kinetics Books; 1991:44-45.

12. Goodman AG, Limbird LE, Hardman JG (ed.). Goodman \& Gilman. As bases farmacológicas da terapêutica. 10. ed. Rio de Janeiro: McGraw-Hill, 2005.

13. Oliveira AF, Valente JG, Leite IC. Aspectos da mortalidade atribuível ao tabaco: revisão sistemática. Rev Saúde Pública. 2008; 42(2):335-45.

14. Ferro CRC, Oliveira DC, Guerra FFG, Lucena AJ, Nunes FP, Ortiz ST et al. Prevalence of and risk factors for combined coronary artery disease and aortic aneurisma. Arq Bras Cardiol. 2007; 88(1):40-44.

15. Gonçalves S, Hardt JR, Silva ASS, Haas P. Hipertensão arterial e a importância da atividade física. Estud Biol 2007; 29(67):205-13.

16. Matavelli IS, Judice ELD, Mataveli R, Hunger MS Martelli A. Hipertensão arterial sistêmica e a prática regular de exercícios físicos como forma de controle: Revisão de Literatura. R bras ci Saúde. 2014; 18(4):359-66.

17. Martelli A. Potencial da prática de exercícios físicos regulares como método não farmacológico no controle da Hipertensão Arterial Sistêmica. Desenvolvimento Pessoal. 2013; 3(2):39-51

18. Barreto-Filho JAS, Krieger JE. Genética e hipertensão arterial: conhecimento aplicado à prática clínica. Rev Soc Cardiol Estado de São Paulo. 2003; 13(1):46-55.

19. Bourbon M. Fatores genéticos e a doença cardiovascular. Rev Port Cardiol. 2008; 27(12):1559-63.

20. Chen CC, Shiu LJ, Li YL, Tung KY, Chan KY, Yeh CJ et al. Shift work and arteriosclerosis risk in professional bus drivers. Ann Epidemiol. 2010; 20(1):60-66.

21. Faria BK, Amorin G, Vancea DMM. Perfil alimentar antropométrico dos motoristas de ônibus da empresa de transportes coletivo Jotur/ Palhoça - SC. RBONE. 2007; $1(1): 11-20$
22. Oliveira RAR, Moreira OC, Andrade Neto F, Amorim W, Costa EG, Marins JCB. Prevalência de sobrepeso e obesidade em professores da Universidade Federal de Viçosa. Fisioter mov. 2011; 24(4):603-12.

23. Cavagioni LC, Bensenõr IM, Halpern A, Pierin AMG. Síndrome metabólica em motoristas profissionais de transporte de cargas da rodovia BR-116 no trecho Paulista-Régis Bittencourt. Arq Bras Endocrinol Metab. 2008; 52(6): 1015-23

24. Masson VA, Monteiro MI. Estilo de vida, aspectos de saúde e trabalho de motoristas de caminhão Estilo de vida, aspectos de saúde e trabalho de motoristas de caminhão Rev Bras Enferm. 2010; 63(4):533-40.

25. Martelli A. Estenose da artéria renal e o desenvolvimento da hipertensão renovascular. Rev Fac Ciênc Méd Sorocaba. 2014; 16(2):59-64.

26. Moraes GN, Fayh APT. Avaliação nutricional e fatores de risco cardiovascular em motoristas de transporte coletivo urbano. Cad. Saúde Colet. 2011; 19(3):334-40.

27. Neri M, Soares WL, Soares C. Condições de saúde no setor de transporte rodoviário de cargas e de passageiros: um estudo baseado na Pesquisa Nacional por Amostra de Domicílios. Cad Saúde Pública. 2005; 21(4):1107-23.

\section{CONFLITO DE INTERESSES}

Os autores declaram não haver conflitos de interesse.

\section{AUTOR PARA CORRESPONDÊNCIA}

\section{Anderson Martelli}

martellibio@hotmail.com

Submetido em 30/05/2018 Aceito em 27/06/2018 